\title{
Premature Ejaculation and Its Pharmacological Treatment
}

\author{
Kim M. Drasa ${ }^{1}$, Jr. Vaso Vasili ${ }^{2}$ \\ Central Polyclinic, Tirana, Albania
}

\begin{abstract}
Introduction and objective: Ejaculatory dysfunction, erectile dysfunction (ED), and decreased libido are the major components of male sexual dysfunction. Premature Ejaculation (PE) however, is actually more prevalent, involving up to $31 \%$ of men aged 18-59 years (1). PE is more prevalent in young men and in men who lack sexual experience and frequency. Our objectives were to assess new avenues in the PE and its Pharmacological Treatment. Material and Methods: $A$ MedLine search was performed for perreviewed articles on Pharmacological Treatment in managing of Premature Ejaculation. Results: Eight manuscripts that examined pharmacological treatment were retrieved. Three studies used drugs as monotherapy and two used it in combination with serotonin selective reuptake inhibitor (SSRI). Two preclinical and early clinical studies with oxytocin antagonists are in progress, but results are still to be released. One may also postulate that dopaminergic drugs interacting with selected receptor subtypes may have potential in this indication. Conclusion: Encouraging evidence supports the role of phosphodiesterase type 5 inhibitors (PDE 5 - Is) in the treatment of PE. SSRIs are one the main categories of pharmacological agents that have been evaluated for PE therapy. Furthermore, in delaying ejaculation, there are a variety of other central neurotransmitters that could be targeted. My feeling is that pharmacological research is still in its infancy in this domain.
\end{abstract}

Keywords: ejaculation, premature, IELT

\section{Prevalence of Premature Ejaculation}

Ejaculatory dysfunction, erectile dysfunction (ED), and decreased libido are the major components of male sexual dysfunction. The introduction of oral pharmacologic therapy for $\mathrm{ED}$ has focused much attention on this pathology compared to others. Premature ejaculation (PE) however, is actually more prevalent, involving up to $31 \%$ of men aged $18-59$ [1], and is considered to be most common among male sexual disorders. PE is more prevalent in young men and in men who lack sexual experience and frequency. The risk of PE does not vary significantly with age, and men with PE are at risk for suffering from psychological disturbances, such as depression and anxiety [2].

\section{PE definitions}

There is no universally accepted definition of PE. The $4^{\text {th }}$ edition of Diagnostic and Statistical Manual of mental Disorders (DSM-IV) defines PE as "persistent or recurrent onset of orgasm and ejaculation with minimal stimulation before the person wishes it" which causes "marked distress or interpersonal difficulty" [3]. This definition consists of four elements of PE: ejaculatory latency, control, distress, and sexual satisfaction. DSM-IV divides PE into lifelong PE, which had been present from the onset of sexual life, and acquired $\mathrm{PE}$, in which the dysfunction developed after a period of normal sexual function. PE can be further divided into global (regardless of partner or situation) and situational (only with certain partners or circumstances). Some authors suggested defining $\mathrm{PE}$ as a syndrome, rather than a complaint, comprising quantification of the ejaculation time, inability of ejaculatory control, and a description of severity of PE in terms of psychological distress [4-5]. Still, the absence of an agreed on definition causes wide variations in patient selection for studies on PE.

\section{Physiology of ejaculation and PE Pathophysiology}

The physiology of ejaculation is still not clearly understood. Ejaculation involves three stages: emission, ejection, and orgasm. Emission consists of contractions of seminal vesicles (SVs) and prostate with expulsion of sperm and seminal fluid into the posterior urethra and is mediated by sympathetic nerves (T10-L2). Ejection is mediated by somatic nerves (S24) and involves pulsatile contractions of the bulbocavernosus and pelvic floor muscles together with relaxation of the external urinary sphincter.

Ejaculation is a complex integrationof actions that occur in the central and peripheral nervous systems. Structures in the central nervous system (CNS) that are involved in ejaculation include the medial preoptic area (MPOA), nucleus paragigantocellularis (nPGi), striaterminalis, amygdala, and thalamus. Whereas the MPOA is involved in stimulation of emission and ejection, the nPGi has an inhibitory influence. Descending serotonergic pathways from the nPGi to the lumbosacral motor nuclei inhibit ejaculation. The MPOA can inhibit the nPGi, which results in ejaculation [6].

Many neurotransmitters are involved in the control of ejaculation, including norepinephrine, serotonin, acetylcholine, oxytocin, y-aminobutyric acid (GABA), and nitric oxide (NO) [7]. Serotonin (5-hydroxytryptamine (5HT) has an inhibitory effect on ejaculation [8]. The serotonin receptors 5-HT $1 \mathrm{a}$ and 5-HT2c were associated with ejaculation (9). 5-HT $1_{\mathrm{a}} \mathrm{a}$ is presynaptic autoreceptor that inhibits serotonin secretion and thus may stimulate ejaculation, whereas the 5-HT2c receptor is postsynaptic and its activation is related to inhibition of ejaculation [9-10], NO has a central effect, mostly in the MPOA, in promoting penile erection, and it may inhibit seminal emission [11]. 


\section{International Journal of Science and Research (IJSR) \\ ISSN (Online): 2319-7064}

Index Copernicus Value (2013): 6.14 | Impact Factor (2014): 5.611

Given the obscurity of ejaculation physiology, it is not surprising that $\mathrm{PE}$ pathophysiology is equally poorly understood. Historically, PE was considered as being a problem with psychological causes, such as anxiety, early sexual experiences, sexual conditioning, and sexual technique [12]. It was speculated that anxiety activates sympathetic output and thus leads the earlier emission and rapid ejaculation [13]. Currently, a speculated neurogenic cause is either a hyposensitivity of 5-HT2c receptors or a hypersensitivity of the 5-HT1a receptors [6]. Other causes might be a hyperexcitable ejaculatory reflex, resulting in faster emission and ejection, prostatic pathologies such as inflammation or infection [14], high fasting serum leptin levels [15] hyperthyroidism [16], and genetic predisposition [17].

History of pharmacological agents that have been used in the treatment of premature ejaculation and Current treatment of Premature Ejaculation and how successful have they been.

Application of topical anaesthetic to reduce the sensitivity of the glans penis is probably the first pharmacological approach used to treat PE. As early as 1943, Bernard Schapiro reported the use of anaesthetic ointment to delay ejaculation. Subsequently, clinical trials have demonstrated the efficacy of two preparations in prolonging ejaculation latency up to 6 to 8 fold. One of these is eutectic mixture of two local anaesthetics, lidocaine and prilocaine (EMLA cream), and the other is an extract from Asian natural herbs (SS cream) [1-2].Adverse events associated with EMLA cream include penile and vaginal numbness; the latter can result inanorgasmia in the female partner unless a condom is used. Local irritation is the most frequent unwanted effect reported with SS cream. The topical treatment of PE has been given a Grade A recommendation by the International Consultation on Sexual Dysfunction in Men and Women held in Paris in 2003[3].

The introduction of antidepressant that enhance central serotonergic function, including monoamine oxidase inhibitors, tricyclic antidepressant, and later, the selective serotonin inhibitors (SSRIs), has resulted in the reporting of side-effects that affect sexual function including delayed ejaculation. In 1973, the first clinical study on the efficacy of the tricyclic antidepressant clomipramine in delaying ejaculation was published [4]. Since then, several authors have demonstrated the potency of daily SSRIs in treating PE in studies with rigorous designs and methodologies [5]. However, chronic exposure to SSRIs can result in the typical adverse events seen in depressive patients and may lead to reduced compliance. More recently the short-acting SSRI dapoxetine given as required to $\mathrm{PE}$ patients have proven effective insignificantly increased ejaculations latency [6]. Treatment of PE with SSRIs has been given Grade A recommendation by International Consultation [3].

Phosphodiesterase -5 inhibitors, well known in the treatment of the erectile dysfunction, have also been tested in PE patients. A few studies have suggested a possible role for sildenafil in PE treatment, especially in combination with an SSRI. A double blind, placebo controlled, multicentre study is still required to validate this pharmacological approach, which currently as a Grade $\mathrm{C}$ recommendation from
International Consultation [3]. In Addition the pharmacological rationale for how phosphodiesterase -5 inhibitors would act in PE has not been elucidated. It is noteworthy that despite strong evidence for the potential of local and systemic treatments to relieve men complaining of $\mathrm{PE}$, there are not drugs currently registered in this indication. SSRIs are one the main categories of pharmacological agents that have been evaluated for PE therapy.

\section{The Mechanism of the Actions of this Drugs}

A great body of evidence indicate that serotonin (5hydroxytryptamine (5-HT)) by acting onbrain postsynaptic receptors exerts inhibitory control on the ejaculatory process. In contrast its action on somatodendriticautoreceptors (5-HT $1 \mathrm{~A}$ subtype) expressed on cell bodies of 5-HTneurons, facilitates ejaculation [5]. Activity of 5-HT neurons is regulated by a negative feedback process initiated upon stimulation of $5-\mathrm{HT}_{1 \mathrm{~A}}$ somatodendriticautoreceptors by released 5-HT. At the level of the nerve terminal, there is further local feedback control by $5-\mathrm{HT}_{1 \mathrm{~B}}$ autoreceptors and activation of these has been shown to reduce synaptic 5-HT concentrations. Another factor that participates in the regulation of 5-HT neuron activity in the 5-HT transporter. This transporter protein, located in the plasma membrane at the level of soma and terminal, removes 5-HT from the extracellular milieu. The main molecular target for SSRIs is the 5-HT transporter. By blocking it, SSRIs cause 5-HT to accumulate in the extracellular space, which leads to higher stimulation of post - synaptic, pre- synaptic and somatodendritic 5-HT receptors. The net result of acute SSRIs administration on 5-HT tone is considered to be insufficient to produce physiological effects, notably delayed ejaculation, since activation on the negative feedback in extracellular 5-HT availability [7]. Prolonged SSRI administration causes desensitisation of somatodendritic and pre-synaptic autoreceptors, rendering the inhibiting autoregulation process less effective. This results in a further increase in 5-HT synaptic levels, which finally translates into physiological effects. These observations serve as a basis for explaining the greater efficacy of chronic SSRI dosing in delaying ejaculation compared with the on-demand regimen [8]. However, this hypothesis has to be re-evaluated, with experimental data to support whether acutely or chronically administered SSRIs (at least fluoxetine and paroxetine) activate serotonergic transmission to the same degree [9]. Recent clinical trials have demonstrated that the efficacy of on-demand dapoxetine, an SSRI structurally related to fluoxetine, in delaying ejaculation is comparable to the chronic SSRIs [6]. In comparison with other SSRIs, dapoxetine has a unique pharmacokinetic profile characterised by rapid absorption and clearance [10]. The mode of action that differentiates dapoxetine from "conventional" SSRIs is still unknown. It has been suggested that dapoxetine gives a very abrupt rise in extracellular 5-HT, which cannot be compensated by autoregulation processes. The post-synaptic 5-HT receptors mediating the effect of increased 5-HT intrasynaptic concentrations on ejaculation are not identified with certainty, although pharmacological investigations have shown $5-\mathrm{HT}_{2 \mathrm{C}}$ and spinal $5-\mathrm{HT}_{1 \mathrm{~B}}$ receptors as being plausible protagonists [11].

\section{Volume 4 Issue 11, November 2015}




\section{International Journal of Science and Research (IJSR)}

ISSN (Online): 2319-7064

Index Copernicus Value (2013): 6.14 | Impact Factor (2014): 5.611

\section{How effective are SSRIs in treating PE and what clinical evidence is there to support their use}

Unquestionably, chronic SSRIs are effective in prolonging ejaculation latency in PE sufferers and non-sufferers. It remains unclear as to whether all SSRIs are equipotent in delaying ejaculation. Paroxetine, fluoxetine and sertraline appear to have similar efficacy in several short-term and longitudinal studies [12-13]. Conversely, a meta-analysis of 35 studies involving daily treatments with an SSRI demonstrated the comparable efficacy of sertraline and fluoxetine in delaying ejaculation, whereas the efficacy of paroxetine was greater than all of the other SSRIs (14).Personal study -"Alleviation of PE by using Sertraline", I had presented as Highlight Poster in ESSM congress,Stamboll, 2014. Conclusion of this study was: Sertraline is better than Dapoxetine for treatment of PE. A limitation in the off-label use of SSRIs in PE is the adverse events associated with chronic exposure, which may be tolerable for the alleviation of depression, but is less acceptable in PE patients. Proposing pharmacotherapy as needed is therefore of particular interest. On the whole, the results of on-demand dosing with SSRIs have been modest. For instance, it has been reported that in a cohort of 30 patients, paroxetine taken on average 5.4 hours before intercourse increased the mean intravaginal ejaculatory latency time (IELT) approximately 1.4-fold [14]. The same drug taken daily for 6 weeks, increased the mean IELT up to 7 -fold [15]. Where stronger claims have been made for ondemand dosing with SSRIs, these have usually been derived from protocols that were methodologically less rigorous [16]. More recently, on-demand depoxetine has been shown to be effective in PE patients when taken 1-3 hours before intercourse, increasing IELT up to 3.5-fold [6].

While most serotonergic agents for PE require chronic administration to maximise the therapeutic effect, never agents are being developed that are effective when used ondemand. These drugs appear to overwhelm the neuronal compensatory mechanisms and it is anticipated that future drugs will combine the best pharmacokinetic properties of short-acting SSRI with deeper understanding of receptors mechanisms involved in serotonergic modulation of ejaculation.

\section{What Newer Groups of Pharmacological Agents are in the Pipeline for Treating PE}

As noted above, the short-acting SSRI dapoxetine (Alza/Johnson \& Johnson) appears promising for treating PE on demand. The future of other such patented agents, UK 390,957 (Pfizer) and BMS 505130 (Bristol-Myers Squibb) is unknown. In view of the autoregulation process of the serotonergic tone, it has been suggested that combining SSRI with a 5-HT1A receptor antagonist may be of interest in improving the onset of action. Interestingly, recent preclinical investigations have shown that this pharmacological approach significantly increases ejaculation latency after a single administration [17-18]. An aerosol delivery of an anesthetic mixture is also under clinical development [19]. There is a scientific rationale for the involvement of oxytocin in the control of ejaculation, although it is still unclear whether this peptide acts centrally and/or peripherally [20]. Preclinicaland early clinical studies with oxytocin antagonists are in progress, but results are still to be released. One may also postulate that dopaminergic drugs interacting with selected receptor subtypes may have potential in this indication [21]. Furthermore, in delaying ejaculation, there are a variety of other central neurotransmitters that could be targeted. My feeling is that pharmacological research is still in its infancy in this domain.

\section{Conclusions}

Intravaginal ejaculatory latency time ( IELT ) is an accepted tool for assessing PE. Currently available IELT measurement methods are not ideal because inherent subjective difficulties in measurement can cause bias in the evaluation on the results. New convenient and efficient technology to measure IELT, which will be less depended on the attention of the sexual partners, would be very useful. In addition, there is a need to perform studies to evaluate correlation between stopwatch-measured IELT and existing questionnaires.

The European and International Society for Sexual Medicine guidelines state that the inclusion criteria for studies on management of PE must ensure that participants have PE and no other sexual dysfunction, such as erectile dysfunction, and that the IELT measurement as a specific entry criterion is not a necessity.

Finally, the inability to reach decisive clinical conclusions is derived from the lack of agreed -on definitions for PE and well- accepted outcome measures to monitor treatment efficacy. The lack of conclusive evidence emphasizes the need for a large population, randomized, double-blind, placebo-controlled study to assess the efficacy of PDE5-Is, SSRI alone, respectivelyor their combination in the management of PE.

\section{References}

[1] Read S. King M, Watson J. Sexual dysfunction in primary medical care: prevalence, characteristics and detection by the general practitioner. J Public Health Med 1997,19: 387-91.

[2] Porst H, Montorsi F, Rosen RC, Gaynor L, Grupe S, Alexander J. The premature Ejaculation Prevalence and Attitudes (PEPA) survey: prevalence, cormobidities, and professional help-seeking. EurUrol 2007,51:816-24

[3] American Psychiatric Association.Diagnostic criteria from DSM-IV-TR. Washington, DC: American Psychiatric Association. 2000

[4] Waldinger MD, Schweitzer DH. Changing paradigms from a historical DSM-III and DSM-IV view towards an evidence based definition of premature ejaculation.Part II: proposals for DSM-V and ICD-11. J Sex Med 2006,3:693-705

[5] Shabsigh R. Diagnosing premature ejaculation: a review. J Sex Med 2006;3(Suppl 4):318-23

[6] Waldinger MD. The neurobiological approach to premature ejaculation. J Urol 2002,168: 2359-67

\section{Volume 4 Issue 11, November 2015}




\section{International Journal of Science and Research (IJSR) \\ ISSN (Online): 2319-7064 \\ Index Copernicus Value (2013): 6.14 | Impact Factor (2014): 5.611}

[7] McMahon CG, Abdo C, IncrocciI, et al. Disorders of orgasmand ejaculation in men. J Sex Med 2004,1;58-65

[8] Guiliano F. Clement P. Serotonin and premature ejaculation:from physiology to patient management. EurUrol 2006;50: 454-66

[9] Waldinger MD, Olivier B. Utility of selective serotonin reuptake inhibitors in premature ejaculation. CurrOpin Investing Drugs 2004;5:743-7

[10] Haensel SM,Mos J, Oliver B,Slob AK. Sex behaviour of male and female Wistars rats affected by serotonin agonist 8-OH-DPAT.Pharmacol BiochemBehav 1991;40:221-8

[11] Sato Y, Horita H, Kurohata T, Adachi H, Tsukamoto T. Effect of the nitric oxide level in the medial preoptic area on male copulatory behaviour in rats. Am J Physiol 1998;274:R243-7

[12]Donatucci CF. Etiology of ejaculation and pathophysiology of premature ejaculation. J Sex Med 2006;3 (Suppl 4):303-8

[13] Kaplan HS, Kohl RN, Pomercy WB, Offit AK, Hogan B. Group treatment of premature ejaculation. ARCH Sex Behav 1974;3:443-52

[14] Screponi E, Carosa E, Di Stasi SM, Pepe M, Carruba G,Jannini EA. Prevalence of chronic prostatitis in men with premature ejaculation. Urology 2001; 58: 198-202

[15] Atmara M, Kuloglu M, Tezcan E, Semercioz A, Ustundag B,Ayar A. Serum Leptin levels in patients with premature ejaculation. Arch Androl 2002; 48.345-50

[16] CaraniC ,Isidori AM, GranataA,et al .Multicenter study on the prevalence of sexual symptoms in male hypo and hyperthyroid patients. J ClinEndocrinolMetab 2005;90:6472-9.

[17] Waldinger MD, Reitschel M, Nothen MM, Hengeveld MW, Oliver B. Familial occurrence of primary premature ejaculation. Psychiatr Genet 1998, 8:37-40.

[18] Mc Mahon CG, Abdo C, Incrocci L, et al. Disorders of orgasm and ejaculation in men.In: Lue TF,Basson R, Rosen R, Guiliano F, Khoury S. Montorsi F, editors, Sexual medicine : Sexual Dysfunction in men and women. Plymouth, UK. Health publication 2004, p.41168.

[19]Eaton H. Clomipramine in the treatment of early ejaculation, J Int Med Res 1973;1:432-4

[20] Guiliano F, Clement P. Serotonin and premature ejaculation: from physiology to patient management. EurUrol 2006,50. 454-66

[21] Pryor JL, Althof SE, Steidle C, et al. Efficacy and tolerability of dapoxetine in treatment of premature ejaculation: an integrated analysis of two double-blind, randomised controlled trials. Lancet 2006, 368:929-37

[22] Guiliano F. 5-HT in premature ejaculation opportunities for therapeutic intervention. Trends Neurosci 2007;30:79-84

[23] Waldinger MD. Oliver B. Utility of selective serotonin reuptake inhibitors in premature ejaculation. Curropin Investing Drugs 2004,18:743-7

[24] De Jong TR. Pattij T. Veening JG. Et al. Citalopram combined with WAY 100635 inhibits ejaculation and ejaculation-related Fosimmunoreactivity. Eur JPharmacol 2005; 509:49-59
[25] Filippi S, Viagnozzi L, Vannelli GB, Ledda F, Forti G, Maggi M, Role of oxytocin in the ejaculatory process. J Endocrinol Invest 2003;26(3 Suppl):82-6. 